\title{
Receptor baseado em cancelamento de interferência para sistema de banco de filtros pré-codificado
}

\author{
Rogério Pereira Junior, Carlos A. Faria da Rocha, Bruno S. Chang e Didier Le Ruyet
}

Resumo-Neste trabalho, avaliamos o cancelamento de interferência iterativa como um método para remover a interferência intrínseca em um sistema de banco de filtros pré-codificado via transformada de Fourier discreta. Este receptor visa possibilitar a utilização de filtros protótipos mais longos melhorando o confinamento espectral e manter o mesmo desempenho de erro. Os resultados numéricos mostram que usando 4 iterações, obtémse desempenho de erro igual ou superior àqueles publicados na literatura que utilizam filtros protótipos com fatores de sobreposição menores. Além disso, o receptor iterativo proposto apresenta uma maior robustez em cenários de alta mobilidade.

Palavras-Chave-Cancelamento de Interferência, Banco de Filtros pré-codificado, Alta Mobilidade, Fator de sobreposição.

\section{INTRODUÇÃO}

O sistema multiportadora baseado em banco de filtros (Filter Bank MultiCarrier - FBMC) é uma alternativa atraente no combate as emissões fora da banda (Out-of-Band - OOB) presentes nos sistemas OFDM (Orthogonal Frequency Division Multiplexing) [1]. Em sistemas FBMC, utiliza-se filtros digitais em cada subportadora para melhorar sua localização no domínio da frequência que consequentemente diminui a emissão OOB [2]. Para isso, a condição de ortogonalidade é relaxada para o campo real, resultando em uma interferência intrínseca concentrada na parte imaginária dos dados transmitidos. No caso de sistemas SISO (Single Input Single Output), essa interferência pode ser eliminada aplicando a modulação OQAM (Offset Quadrature Amplitude Modulation) [3]. No entanto, o uso combinado de FBMC com a técnica MIMO (Multiple-Input Multiple-Output) não é simples devido à perda da ortogonalidade complexa. Portanto, versões précodificadas foram propostas como em [4], [5] para combater essas interferências e restaurar a ortogonalidade total.

Recentemente, os autores em [6] apresentaram um novo método de pré-codificação baseado no espalhamento de uma DFT (discrete Fourier transform) podada combinada com um fator de escala para compensar a interferência do filtro. Este esquema apresenta as vantagens da modulação FBMC e SCFDMA (Single-carrier frequency-division multiple access), com espectro bem localizado, menores emissões OOB, e baixa PAPR (Peak to Average Power Ratio), além de restaurar parcialmente a ortogonalidade complexa. No entanto, essa

Rogério Pereira Junior e Carlos A. Faria da Rocha, Grupo de Pesquisa em Comunicação da Universidade Federal de Santa Catarina, Florianópolis, Brasil, e-mail:rogerio.pereira.junior@posgrad.ufsc.br, carlos.aurelio@ufsc.br; Bruno S. Chang, CPGEI/Departamento de Eletrônica da Universidade Tecnológica Federal do Paraná, Curitiba, Brasil, e-mail:bschang@utfpr.edu.br; Didier Le Ruyet, Conservatoire National des Arts et Métiers, Paris, França, e-mail:leruyet@cnam.fr. Este trabalho foi parcialmente financiado pela Coordenação de Aperfeiçoamento de Pessoal de Nível Superior (CAPES) - Brasil. técnica tem uma limitação em relação ao fator de sobreposição máximo do filtro. Os autores mostram que o fator máximo que pode ser usado para evitar a interferência intrínseca é de aproximadamente 1,5, o que gera emissões OOB maiores em comparação com o FBMC convencional que não apresenta essa restrição em relação ao comprimento do filtro. Em [7] é proposto um novo sistema de banco de filtros baseado também no espalhamento via DFT. O novo esquema utiliza a transmissão em taxa dobrada combinada com uma estratégia de transmissão de dados, sem o uso de OQAM. Além disso, também é introduzido um equalizador realimentado por decisão no formato iterativo (Iterative Block Decision Feedback Equalizer - IB-DFE) para obter melhor desempenho em termos de taxa de erro de bit (Bit Error Rate - BER) e em alguns cenários, trabalhar com maiores fatores de sobreposição de filtro e consequentemente uma melhor localização espectral. Porém, como o projeto do IB-DFE é baseado apenas no conhecimento do canal, o ganho de desempenho em termos de BER diminui significantemente quando fatores de sobreposição da ordem de 4 (caso do filtro Phydyas [8]) são utilizados, visto que neste caso surgem interferências oriundas do filtro. Assim, receptores mais robustos são necessários para minimizar este problema. O uso de técnicas baseadas no cancelamento de interferências pode ser uma abordagem alternativa. Em [9] [10] [11] e [12], são apresentados alguns casos de algoritmos de cancelamento de interferência para o sistema FBMC a fim de mitigar a interferência residual do filtro e de canais com alta mobilidade.

O objetivo deste trabalho é propor um receptor de cancelamento de interferência no formato iterativo (Iterative Interference Cancellation - IIC) para o sistema de banco de filtros pré-codificados via DFT de modo que seja possível a utilização de filtros protótipos mais longos. Desta forma, pode-se obter um bom desempenho de erro e uma redução da emissão OOB, aproveitando ao máximo os recursos disponíveis. Além disso, são apresentados resultados de simulações que comparam o desempenho em termos de BER do equalizador utilizando cancelamento de interferências com o esquema IB-DFE proposto em [7]. Os resultados mostraram que o esquema utilizando cancelamento de interferências é mais eficiente que o IB-DFE tanto utilizando filtros protótipos com fatores de sobreposição maiores quanto para canais com forte espalhamento Doppler.

$\mathrm{O}$ artigo está organizado da seguinte forma: A Seção II descreve o modelo do sistema de Banco de Filtros précodificado. A Seção III apresenta o algoritmo de cancelamento de interferência proposto. Por fim, os resultados da simulação são apresentados na Seção IV e a Seção V conclui o artigo.

Notação: os vetores e matrizes são descritos respectivamente 
por letras minúsculas e letras maiúsculas em negrito. A $i$-ésima linha e o $j$-ésima elemento da coluna da matriz $\mathbf{M}$ é denotado por $[\mathbf{M}]_{i, j}$. O sobrescrito $(.)^{H}$ denota a operação Hermitiana, a matriz de identidade de tamanho $N \times N$ é denotada por $\mathbf{I}_{N}$, $\operatorname{diag}(\mathbf{M})$ produz uma nova matriz diagonal com os mesmos elementos da diagonal principal de $\mathbf{M}$ e $\operatorname{diag}(\mathbf{m})$ representa a geração de uma matriz diagonal dos elementos do vetor $\mathbf{m}$.

\section{Sistema de BAnCo DE Filtros PRÉ-CODifiCAdo}

Considere $x_{l, k}$ um símbolo complexo transmitido na $l$-ésima subportadora no instante de tempo $k$. A transmissão deste simbolo por um banco de filtros pode ser expresso em tempo discreto por [3]

$$
s[m]=\sum_{l=0}^{L-1} \sum_{k=0}^{K-1} x_{l, k} g_{l, k}[m]
$$

onde $g_{l, k}[m]$ é dado por

$$
g_{l, k}[m]=g[m-k L / 2] e^{j \frac{2 \pi l}{L}(m-k L / 2)}
$$

e é essencialmente uma versão deslocada no tempo e frequência do filtro protótipo $g[\mathrm{~m}]$ de comprimento $O L$, onde $O$ denota o fator de sobreposição do filtro, enquanto $K$ indica o número de símbolos no tempo com $L$ subportadoras cada.

Para restabelecer a ortogonalidade complexa, os sistemas propostos em [6], [7] usam um método de pré-codificação, composto por uma transformada de Fourier combinada com um estágio de pré-compensação da interferência intrínseca do filtro. No caso do último esquema, $L / 2$ símbolos complexos são multiplicados por um fator de escala e são inseridos nas $L / 4$ primeiras e últimas posições de uma DFT de comprimento $L$. Os valores intermediários são zeros, conforme mostrado na Figura 1.

Este sistema de banco de filtros pode ser caracterizado por uma estrutura de blocos com $K$ símbolos no tempo, em que cada símbolo possui $L$ subportadoras. Assim, considerando uma estrutura matricial, o vetor de dados transmitidos no $k$ ésimo instante de tempo $\mathbf{x}_{k} \in C^{L \times 1}$ é expresso por

$$
\mathbf{x}_{k}=\mathbf{C}_{f} \mathbf{a}_{k}
$$

onde $\mathbf{a}_{k} \in C^{L \times 1}$ é um vetor com $L / 2$ símbolos complexos de dados e $\mathbf{C}_{f} \in C^{L \times L}$ é a matriz de pré-codificação, dada por

$$
\mathbf{C}_{f}=\mathbf{W}_{L} \operatorname{diag}\{\tilde{\mathbf{b}}\},
$$

onde $\mathbf{W}_{L} \in C^{L \times L}$ é a matriz DFT normalizada e $\tilde{\mathbf{b}}$ é o fator de escala do filtro de tamanho $L \times 1$, sendo $L / 2$ coeficientes de compensação nas posições de acordo com a estratégia de transmissão definida para $\mathbf{a}_{k}$ e as posições restantes igual a zero. Desta forma, a $i$-ésima posição do vetor $\tilde{\mathbf{b}}$ pode ser expressa da seguinte forma

$$
[\tilde{\mathbf{b}}]_{i}=\sqrt{\frac{1}{[\tilde{\mathbf{C}}]_{i, i}}}, \text { para } i=\left\{1, \ldots, \frac{L}{4} ; L-\frac{L}{4}, \ldots, L\right\},
$$

onde

$$
\tilde{\mathbf{C}}=\mathbf{W}_{L}^{H} \mathbf{G}_{k}^{H} \mathbf{G}_{k} \mathbf{W}_{L},
$$

com $\mathbf{G}_{k}$ representando a matriz do filtro. Para definição da matriz $\mathbf{G}_{k}$ a versão deslocada do filtro (equação (2)) é amostrada gerando o vetor $\mathbf{g}_{l, k} \in C^{N \times 1}$ com $N$ amostras [6]. Através dessa amostragem defini-se a matriz de transmissão $\mathbf{G}_{k}$ no tempo $k$ por $\mathbf{G}_{k}=\left[\mathbf{g}_{1, k}, \ldots, \mathbf{g}_{L, k},\right] \in C^{N \times L}$. Empilhando todos os vetores do pulso, temos a matriz geral de transmissão $\mathbf{G}=\left[\mathbf{G}_{1}, \ldots, \mathbf{G}_{K}\right] \in C^{N \times L K}$. Usando esta notação, o sinal a ser transmitido dado pela em (1) pode ser expresso no formato matricial pelo vetor $\mathbf{s} \in C^{N \times 1}$ como [6]

$$
\mathbf{s}=\mathbf{G} \mathbf{x}
$$

$\mathrm{O}$ vetor dos símbolos de dados $\mathbf{x} \in C^{L K \times 1}$, é obtido através da serialização do vetor $\mathbf{x}_{k}$ para todo $k$, isto é, $\mathbf{x}=\operatorname{vec}\left\{\left[\mathbf{x}_{1}, \ldots, \mathbf{x}_{k}\right]\right\}$. Na prática, (7) pode ser implementada de forma eficiente através do uso de uma DFT inversa (Inverse DFT - IDFT) combinado com uma rede polifásica (Polyphase Network - PPN) [3]. Uma abordagem matricial nesta configuração que simplifica o entendimento do processo de compensação por $\mathbf{C}_{f}$ pode ser encontrada em [7].

A transmissão do vetor $\mathbf{s}$ é realizada através de um canal multipercurso variável no tempo utilizando a matriz de convolução $\mathbf{H} \in C^{N \times N}$. O vetor recebido $\mathbf{y} \in C^{L K \times 1}$ é obtido multiplicando as amostras transmitidas pelo canal por $\mathbf{G}^{H}$, resultando na expressão

$$
\mathbf{y}=\mathbf{D} \mathbf{x}+\mathbf{G}^{H} \mathbf{n}
$$

sendo a matriz $\mathbf{D} \in C^{L K \times L K}$ dada por

$$
\mathbf{D}=\mathbf{G}^{H} \mathbf{H G}
$$

e $\mathbf{n}$ representa as amostras do ruído gaussiano branco de média nula e variância $\sigma_{n}^{2}$. A detecção do símbolo $\tilde{\mathbf{a}}_{k} \in C^{L \times 1}$ é obtida após a equalização de 1 coeficiente por $\mathbf{e}_{k} \in C^{L \times 1}$ seguido pela decodificação de acordo com

$$
\tilde{\mathbf{a}}_{k}=\mathbf{C}_{f}^{H} \operatorname{diag}\left\{\mathbf{e}_{k}\right\} \mathbf{y}_{k},
$$

Para um fator de sobreposição igual ou menor do que 1,5 , esta técnica compensa a interferência intrínseca do filtro protótipo e recupera a condição de ortogonalidade. Porém, a limitação do valor do fator de sobreposição impede a utilização de filtros mais bem localizados no domínio da frequência e, portanto, um melhor confinamento espectral. Caso o valor de sobreposição seja maior do que 1,5 surgem interferências que a codificação não consegue mais eliminar por completo e técnicas mais robustas de recepção são necessárias para uma melhor detecção dos símbolos.

\section{CANCELADOR DE INTERFERÊNCIA ITERATIVO}

Geralmente, equalizadores de 1 coeficiente calculados através dos critérios MMSE (Minimum Mean Square Error) ou de Forçagem a Zero (Zero Forcing - ZF) são usados para compensar a interferência oriundas do canal de comunicação. No entanto, em um sistema baseado em banco de filtros sem o uso de um prefixo cíclico, em certas ocasiões este equalizador não é suficiente, especialmente em cenários de alta mobilidade com altos valores de SNR (Signal-to-Noise Ratio). Em [7], foi adotado um equalizador iterativo mais robusto que consegue minimizar ainda mais essas interferências. Como 

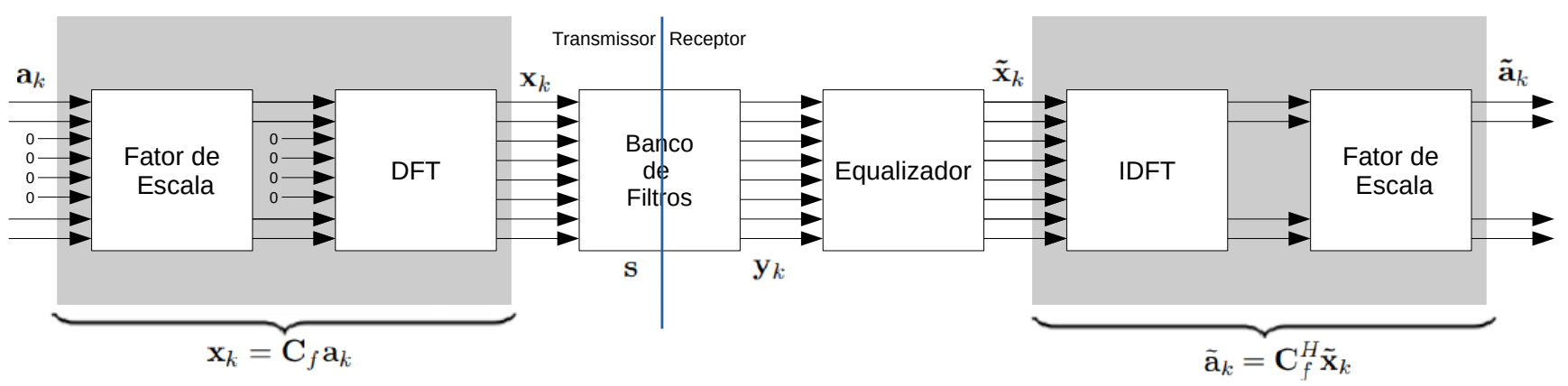

Fig. 1: Diagrama de blocos do sistema de banco de filtros pré-codificado via DFT.

este equalizador compensa fortemente a interferência do canal, é possível aumentar o fator de sobreposição do filtro em algumas situações sem perda significativa de desempenho, mesmo que não resolva a interferência do filtro. Assim, é mais interessante um receptor que lide com ambas as interferências.

Analisando (10), a relação entrada-saída de todo o sistema de transmissão definida pelo vetor $\tilde{\mathbf{a}} \in C^{L K \times 1}$ pode ser modelada por

$$
\tilde{\mathbf{a}}=\mathbf{C}^{H} \mathbf{E G}{ }^{H} \mathbf{H G C a}+\mathbf{C}^{H} \mathbf{E G}{ }^{H} \mathbf{n}
$$

onde $\tilde{\mathbf{a}} \in C^{L K \times 1}$ fornece uma estimativa dos símbolos de dados transmitidos $\mathbf{a}=\operatorname{vec}\left\{\left[\mathbf{a}_{1}, \ldots, \mathbf{a}_{k}\right]\right\} \in C^{L K \times 1} . \mathbf{C} \in$ $C^{L K \times L K}$ e $\mathbf{E} \in C^{L K \times L K}$ são dados, respectivamente, por

$$
\mathbf{C}=\mathbf{I}_{K} \otimes \mathbf{C}_{f}
$$

$\otimes$ é o produto de Kronecker e

$$
\mathbf{E}=\operatorname{diag}\left\{\operatorname{vec}\left\{\left[\mathbf{e}_{1}, \ldots, \mathbf{e}_{k}\right]\right\}\right\}=\operatorname{diag}\{\mathbf{D}\}^{-1}
$$

representa os coeficientes do equalizador, visto que a diagonal principal de $\mathbf{D}$ descreve os coeficientes do canal, uma vez que em uma variedade de cenários práticos, os elementos fora da diagonal, são pequenos e dominados pelo ruído [10]. A Figura 2 apresenta um esquema simples, mas eficaz, de cancelamento de interferência em um formato iterativo que trata tanto os problemas das interferências provocadas pelo canal quanto daquela do filtro protótipo. Para valores do fator

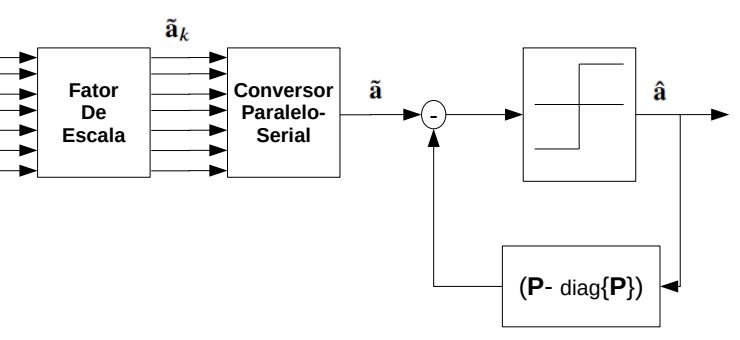

Fig. 2: Diagrama de blocos do receptor IIC.

de sobreposição acima de 1,5 , os símbolos de dados recebidos são corrompidos pela interferência intrínseca do filtro que complica a detecção correta dos dados. Assim, esquemas de detecção com estimativa e cancelamento de interferência nem sempre são eficazes devido à propagação do erro. Portanto, o desafio é mitigar a propagação do erro por meio de iterações, ou seja, melhorar a confiabilidade dos símbolos detectados a cada iteração. A ideia básica do esquema proposto é, primeiro, o equalizador ZF, seguido pela pós-codificação, fornece estimativas provisórias dos vetores de dados. Então, com base nessas estimativas, o cancelador de interferência calcula uma estimativa da interferência que deve ser minimizada.

Através da definição da relação entrada-saída do sistema em (11), a contribuição da interferência vinda do filtro e do canal (sem o ruído) pode ser definida pela matriz $\mathbf{P} \in$ $C^{L K \times L K}$ expressa por

$$
\mathbf{P}=\mathbf{C}^{H} \mathbf{E G}^{H} \mathbf{H G C}
$$

A interferência é descrita pelos elementos fora da diagonal principal da matriz $\mathbf{P}$, pois se $O=1.5$, temos $\mathbf{C}^{H} \mathbf{G}{ }^{H} \mathbf{G C} \approx$ $\mathbf{I}_{L K / 2}$. Para reduzir o nível de interferências e melhorar o desempenho, os termos indesejados na matriz $\mathbf{P}$ devem ser removidos. Assim, de forma semelhante ao sugerido em [13] para um sistema OFDM podemos cancelar a interferência na entrada do quantizador considerando a $i$-ésima iteração por

$$
\tilde{\mathbf{a}}^{i+1}=\tilde{\mathbf{a}}-(\mathbf{P}-\operatorname{diag}\{\mathbf{P}\}) \hat{\mathbf{a}}^{i}
$$

Deve-se notar que os $L / 2$ símbolos são quantizados definindo o vetor â; portanto, é necessário gerar a mesma estratégia de alocação dos dados feita no transmissor para passar pelo cancelador de interferência. Vale ressaltar que $(\mathbf{P}-\operatorname{diag}\{\mathbf{P}\})$ são os termos de interferência que são subtraídos através do processo iterativo, trazendo maior confiabilidade aos símbolos detectados. Ou seja, o receptor subtrai os termos de interferência dos símbolos de dados e o processo é repetido iterativamente até que um número predefinido de iterações seja satisfeito. Considerando apenas a interferência do filtro, ou seja, $\mathbf{H}=\mathbf{I}_{N}$, a Figura 3 mostra a representação da interferência dentro e fora da diagonal da matriz $\mathbf{P}$ para fatores de sobreposição iguais e maiores que 1,5 . Observe que para $O=1.5$, a interferência fora da diagonal é parcialmente suprimida pela codificação via DFT mais o fator de escala. Quando este limite é excedido, interferências aparecem e usando o cancelador de interferência para removê-las, temos uma estimativa mais limpa e confiável.

A ideia do IIC é reduzir ainda mais a influência da interferência residual do filtro e aumentar a robustez em cenários de alta mobilidade. A remoção dessas interferências pode permitir a aplicação de algumas técnicas convencionais MIMO usando filtros mais longos. Além disso, pelas características de baixa 

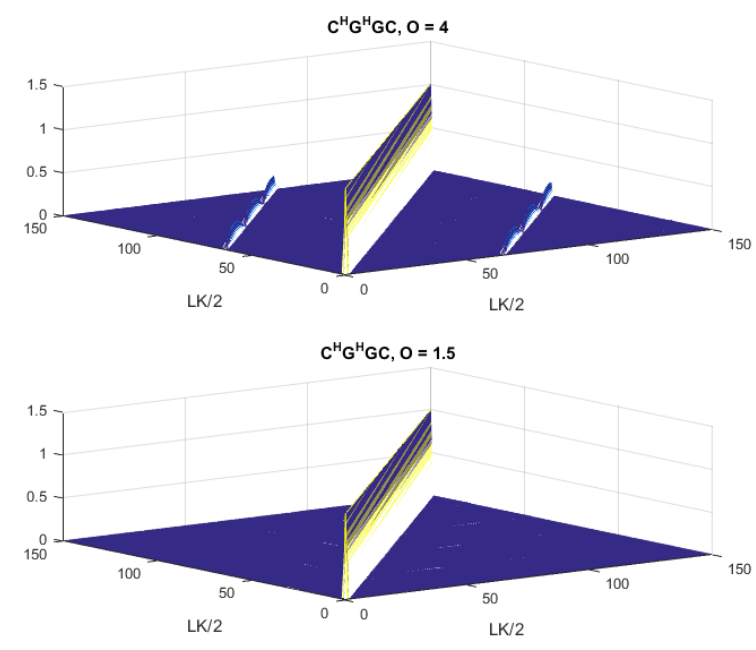

Fig. 3: Representação de termos interferentes em $\mathbf{C}^{H} \mathbf{G}{ }^{H} \mathbf{G}$.

PAPR devido à sua pré-codificação via DFT, é interessante utilizar o IIC no uplink, pois no receptor a complexidade estará concentrada na estação base.

\section{Resultados de SimulaçÃo}

Nesta seção, são apresentados os resultados de simulação para sistema de banco de filtros pré-codificados via DFT com o receptor proposto em termos de BER. O sinal transmitido é afetado por um canal com desvanecimento do tipo Rayleigh. Considera-se o perfeito conhecimento do canal pelo receptor e utiliza-se o modelo TDL (Tapped Delay Line) - A [14]. Em futuros sistemas sem fio espera-se que a formatação dos feixes e o tamanho pequeno das célula levem a um espalhamento de atraso muito mais curto. Um espaçamento entre subportadoras de $15 \mathrm{kHz}$ foi considerado.

Para o cancelamento de interferência, 4 iterações foram consideradas, uma vez que o ganho de desempenho não é significativo acima deste valor e também para manter a complexidade computacional baixa. Para efeito de comparação, também descrevemos o desempenho do sistema com o equalizador IB-DFE visto em [7] e pelo Genie-Aided $\left(\hat{\mathbf{a}}^{i}=\mathbf{a}\right.$ em (15)). Desta forma, tem-se o desempenho máximo que o receptor proposto consegue obter. No caso das curvas com $O=1,5$, o filtro Hermite truncado é usado e para os outros casos, foi adotado o filtro Phydyas com $O=4$. Ambos os filtros são detalhados respectivamente em [8] e [6].

A Figura 4 apresenta a BER não codificada para a modulação 16-QAM, $L=32, K=16$ e velocidade de $0 \mathrm{~km} / \mathrm{h}$. Observa-se claramente o ganho de desempenho da técnica proposta quando comparada ao caso MMSE (IB-DFE com uma iteração) com $O=4$. Note que as curvas obtidas com uma e quatro iterações do IIC com $O=4$ são semelhantes àquelas obtidas com $O=1,5$ e "Genie-Aided", indicando que a interferência (elementos fora da diagonal principal da matriz $\mathbf{P}$ ) não é elevada. Deve-se notar que para este caso sem mobilidade, o equalizador de um coeficiente é suficiente e eficaz para cancelar a interferência induzida pelo canal. Assim, quando temos o receptor proposto com $O=1,5$ não há

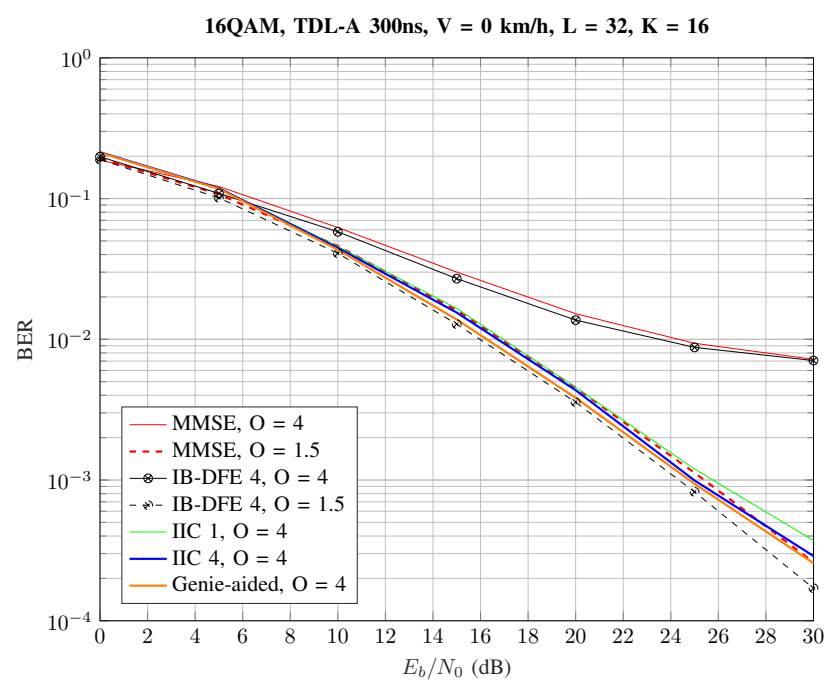

Fig. 4: BER para 16-QAM, TDL-A e velocidade de $0 \mathrm{~km} / \mathrm{h}$.

melhora de desempenho, uma vez que a interferência do filtro é compensada quase que completamente pelo sistema. Quando comparado com o equalizador IB-DFE o esquema proposto tem desempenho bastante superior para $O=4$, devido à remoção iterativa das interferências do sistema.

Os resultados apresentados na Figura 5 consideram o mesmo cenário, mas, agora com velocidade de $300 \mathrm{~km} / \mathrm{h}$. Neste caso, o canal é de alta mobilidade e o desempenho de

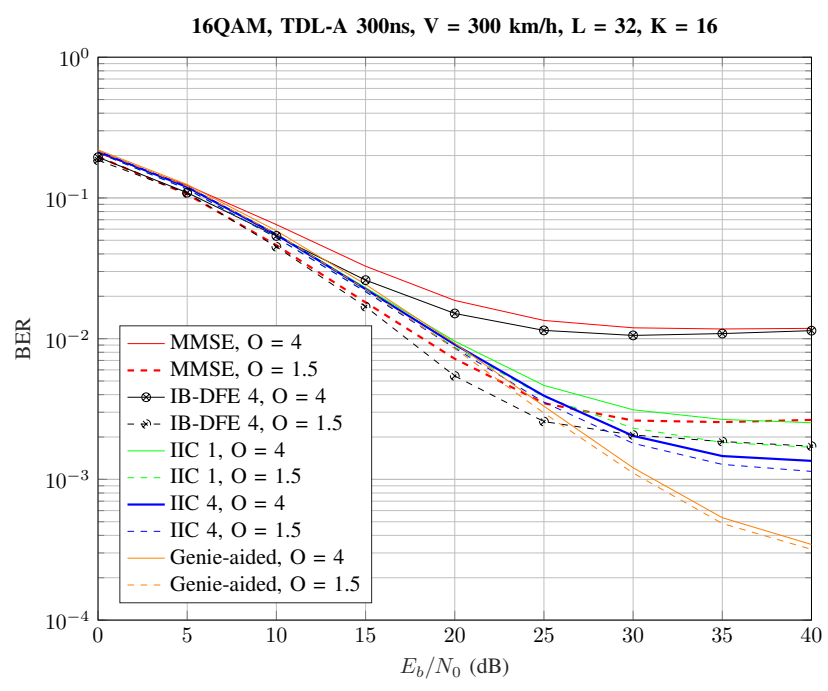

Fig. 5: BER para 16-QAM, canal TDL-A e velocidade de $300 \mathrm{~km} / \mathrm{h}$.

erro é afetado, pois agora as interferências são provenientes não só do filtro mas também no canal variante no tempo. Note que, neste caso, o ganho de desempenho do receptor proposto é significativo, mesmo para $O=1,5$. Novamente, para o caso genie-aided, o receptor iterativo obtém melhor desempenho desta vez, em altas SNRs. Deve-se notar que o pior desempenho em baixas SNRs do receptor proposto é devido ao uso do equalizador $\mathrm{ZF}$ antes do processo de cancelamento. Ao usar o equalizador MMSE, essa diferença é atenuada. Neste cenário o IB-DFE com $O=4$ tem seu desempenho severamente afetado pelas interferências, tornando assim o receptor proposto uma 
alternativa com melhor desempenho.

As Figuras 6 e 7 apresentam resultados para os mesmos cenários das Figuras 4 e 5, porém agora para a modulação 64-QAM. Observe novamente que o receptor consegue obter

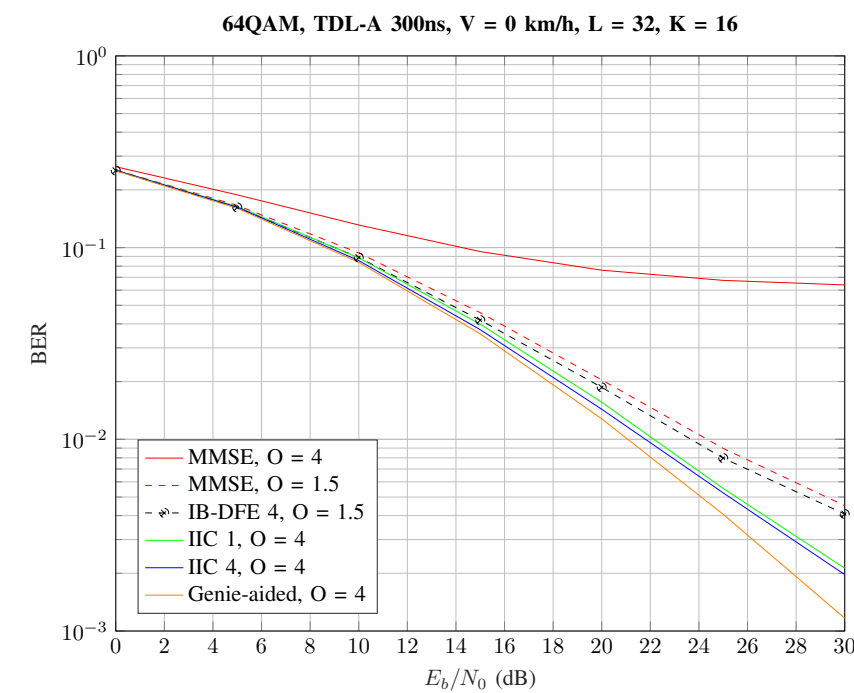

Fig. 6: BER para 64-QAM, canal TDL-A e velocidade de $0 \mathrm{~km} / \mathrm{h}$.

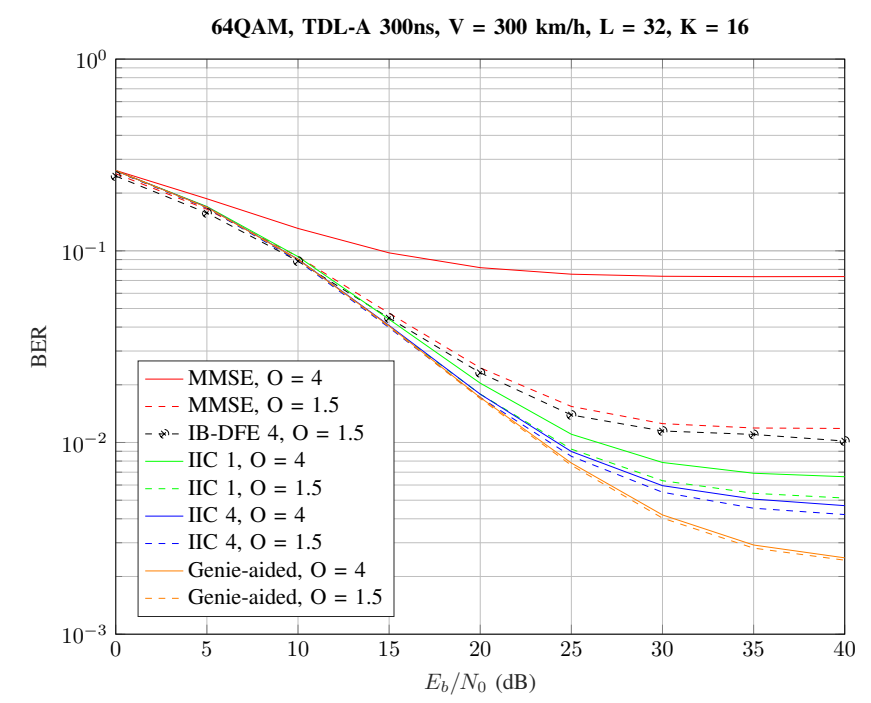

Fig. 7: BER para 64-QAM, canal TDL-A e velocidade de $300 \mathrm{~km} / \mathrm{h}$.

um bom desempenho mesmo usando um filtro mais longo, com um desempenho semelhante ao caso $O=1.5$. Além disso, no cenário de alta mobilidade o ganho de desempenho é ainda mais considerável e novamente, no modo genie-aided um alto ganho é obtido em comparação com o mesmo receptor no caso sem mobilidade. Como este cenário é mais sensível às interferências, mesmo com $O=1,5$ o IB-DFE tem pior desempenho que o receptor proposto com $O=4$, devido à remoção iterativa das interferências realizada.

Atualmente, é fundamental que os sistemas de comunicação tenham alta tolerância a interferências e robustez contra erros de sincronização no tempo e frequência. Neste contexto, quanto maior o fator de sobreposição do filtro, melhor será a localização espectral (reduzir $O$ aumenta as emissões
OOB). Como esse aumento causa interferência no sistema, o receptor proposto nos permite tratar essas interferências, validando assim a abordagem iterativa apresentada. O esquema de cancelamento de interferência é responsável por aumentar o desempenho do sistema, principalmente em ambientes de alta mobilidade.

\section{CONCLUSÃO}

No sistema de banco de filtros pré-codificado via DFT, o uso de fatores de sobreposição acima de 1,5 suprime a ortogonalidade complexa e aumenta a interferência no sistema. A utilização de técnicas de cancelamento de interferência é utilizada para minimizar esses efeitos. Neste artigo, apresentamos um esquema de cancelamento de interferência iterativo que nos permite estender o fator de sobreposição do filtro acima de 1,5 , obtendo bom confinamento espectral e bom desempenho de erro. Este receptor apresentou bom desempenho comparado a um equalizador iterativo mais robusto no tratamento das interferências do canal. Além disso, o esquema mostrou-se robusto em cenários de alta mobilidade.

\section{REFERÊNCIAS}

[1] B. Farhang-Boroujeny, "OFDM versus filter bank multicarrier," IEEE signal processing magazine, vol. 28, no. 3, pp. 92-112, 2011.

[2] M. Schellmann, Z. Zhao, H. Lin, P. Siohan, N. Rajatheva, V. Luecken, and A. Ishaque, "FBMC-based air interface for $5 \mathrm{~g}$ mobile: Challenges and proposed solutions," in 2014 9th International Conference on Cognitive Radio Oriented Wireless Networks and Communications (CROWNCOM). IEEE, 2014, pp. 102-107.

[3] P. Siohan, C. Siclet, and N. Lacaille, "Analysis and design of OFDM/OQAM systems based on filterbank theory," IEEE transactions on signal processing, vol. 50, no. 5, pp. 1170-1183, 2002.

[4] R. Zakaria and D. Le Ruyet, "A novel filter-bank multicarrier scheme to mitigate the intrinsic interference: Application to MIMO systems," IEEE Transactions on Wireless Communications, vol. 11, no. 3, pp. 1112-1123, 2012.

[5] D. Demmer, R. Zakaria, R. Gerzaguet, J.-B. Doré, and D. Le Ruyet, "Study of OFDM precoded filter-bank waveforms," IEEE Transactions on Wireless Communications, vol. 18, no. 6, pp. 2889-2902, 2018.

[6] R. Nissel and M. Rupp, "Pruned DFT-spread FBMC: low PAPR, low latency, high spectral efficiency," IEEE Transactions on Communications, vol. 66, no. 10, pp. 4811-4825, 2018.

[7] R. Pereira, C. A. da Rocha, B. S. Chang, and D. Le Ruyet, "A novel DFT precoded filter bank system with iterative equalization," IEEE Wireless Communications Letters, 2020.

[8] M. Bellanger, D. Le Ruyet, D. Roviras, M. Terré, J. Nossek, L. Baltar, Q. Bai, D. Waldhauser, M. Renfors, T. Ihalainen et al., "FBMC physical layer: a primer," Phydyas, vol. 25, no. 4, pp. 7-10, 2010.

[9] B. Kwon, S. Kim, D. Jeon, and S. Lee, "Iterative interference cancellation and channel estimation in evolved multimedia broadcast multicast system using filter-bank multicarrier-quadrature amplitude modulation,' IEEE Transactions on Broadcasting, vol. 62, no. 4, pp. 864-875, 2016.

[10] R. Nissel, M. Rupp, and R. Marsalek, "FBMC-OQAM in doublyselective channels: A new perspective on MMSE equalization," in 2017 IEEE 18th International Workshop on Signal Processing Advances in Wireless Communications (SPAWC). IEEE, 2017, pp. 1-5.

[11] Y. J. Harbi and A. G. Burr, "IIC of the MIMO-FBMC/OQAM system using linear and SIC detection schemes in LTE channel," in 2018 IEEE Wireless Communications and Networking Conference (WCNC). IEEE, 2018, pp. 1-6.

[12] S. Mahama, Y. J. Harbi, A. G. Burr, and D. Grace, "Iterative interference cancellation in FBMC-QAM systems," in 2019 IEEE Wireless Communications and Networking Conference (WCNC). IEEE, 2019, pp. $1-5$.

[13] A. F. Molisch, M. Toeltsch, and S. Vermani, "Iterative methods for cancellation of intercarrier interference in OFDM systems," IEEE Transactions on Vehicular Technology, vol. 56, no. 4, pp. 2158-2167, 2007.

[14] J. Meredith, "Study on channel model for frequency spectrum above 6 GHz," 3GPP TR 38.900, Jun, Tech. Rep., 2016. 